\title{
Hemoptisis en el espectro del edema pulmonar neurogénico
}

\author{
Hemoptysis in the spectrum of neurogenic \\ pulmonary edema
}

\author{
Carlos Alfonso Machado R., MD. (1); Leidy Samara Pinilla Q., MD. ${ }^{(2)}$; HÉctor AndRÉs \\ Castañeda C., MD. ${ }^{(2)}$; LaURa Yasmín Ramírez R., MD. ${ }^{(3)}$; XueYI Chen, EM. ${ }^{(4)}$; Claudia \\ Juliana Patiño O., EM. ${ }^{(4)}$; Silvia Camila Amaya A., EM. ${ }^{(5)}$; AndRea PaOla Ortega G., EM. ${ }^{(5)}$
}

\section{Resumen}

El edema pulmonar neurogénico es un diagnóstico de exclusión, cuya frecuencia de presentación no ha sido establecida, dada la falta de criterios diagnósticos bien validados que permitan realizar un abordaje inicial temprano en el paciente con un evento y/o insulto en el sistema nervioso central. Los dos principales mecanismos fisiopatológicos están dados por: aumento de la permeabilidad capilar y en la vasoconstricción pulmonar y sistémica que permiten la expresión radiológica de patrón de ocupación alveolar difusa con un sin número de diagnósticos diferenciales según el escenario clínico. Dado que el estatus convulsivo ha sido catalogado como la principal causa de edema pulmonar neurogénico; ante la presencia probable de estatus convulsivo inicial con hallazgos inespecíficos de edema pulmonar de etiología no cardiogénico y hemoptisis se expone un caso inusual de edema pulmonar neurogénico con resolución clínica y radiológica al cabo de 48 horas del cese completo de las crisis parciales, siendo esta última medida el principal objetivo de tratamiento en el paciente con dicha condición.

Palabras clave: edema pulmonar, edema pulmonar neurogénico, epilepsia.

\begin{abstract}
Neurogenic pulmonary edema is a diagnosis of exclusion, whose frequency has not been established due to lack of well validated diagnostic critera that could allow for an early initial approach to the patient with an event and/or injury of the central nervous system. The main two pathophysiological mechanisms are given by: (1) increased capillary permeability and (2) pulmonary and systemic vasoconstriction, both of which cause the radiological expression of diffuse alveolar occupation pattern, with countless differential diagnoses according to the clinical scenario. Status epilepticus has been classified as the main cause of neurogenic pulmonary edema. Therefore, the latter shoud be suspected when probable status epilepticus first appears, with unspecific findings of non-cardiogenic pulmonary edema and hemoptysis. We present an unusual case of neurogenic pulmonary edema, with complete clinical and radiological resolution 48 hours after termination of the partial crises. The latter is the main goal of treatment in patients with neurogenic pulmonary edema.
\end{abstract}

Keywords: pulmonary edema, neurogenic pulmonary edema, epilepsy.

\footnotetext{
${ }^{(1)}$ Medicina Interna, Universidad Industrial de Santander UIS, Fellow Neumología Universidad Javeriana. Bogotá, Colombia. ${ }^{(2)}$ Residente de Medicina Interna Segundo año, Universidad Autónoma. Bucaramanga, Colombia.

${ }^{(3)}$ Médica Interna, Universidad Autónoma. Bucaramanga, Colombia.

${ }^{(4)}$ Estudiante de Medicina VIII Semestre, Universidad Autónoma. Bucaramanga, Colombia.

${ }^{(5)}$ Estudiante de Medicina VII Semestre, Universidad Autónoma. Bucaramanga, Colombia.

Correspondencia: Leidy Samara Pinilla Q., correo electrónico: samara_pinilla@ yahoo.es

Recibido: 04/04/17. Aceptado: 30/04/17.
} 


\section{Introducción}

El edema pulmonar neurogénico es un diagnóstico diferencial importante del edema pulmonar cardiogénico, siendo este último el más común. Sin embargo, el edema pulmonar neurogénico es una entidad subdiagnosticada por su poca frecuencia y cuya clave en el abordaje inicial consiste en documentar el factor desencadenante descrito como una lesión del sistema nervioso central, históricamente relacionado con hemorragia subaracnoidea y epilepsia. Se expone el caso de una paciente adulta mayor con antecedentes de epilepsia e hipertensión arterial, con síntomas respiratorios asociados a status convulsivo y ocupación alveolar difusa, inicialmente tratado como neumonía aspirativa, con respuesta clínica favorable y aparición de hemoptisis luego de 48 horas de tratamiento, sin deterioro respiratorio y documentación imagenológica de resolución completa de los hallazgos previos, descartándose otras causas probables como neumonía aspirativa, edema pulmonar cardiogénico, malignidad, tuberculosis pulmonar, entre otras; el edema pulmonar neurogénico fue el diagnóstico más probable por exclusión y el más pertinente para el caso.

\section{Caso}

Paciente de género femenino, de 78 años de edad, quien consultó por cuadro de dos días de evolución caracterizado por crisis parciales complejas con aura somatosensorial autolimitadas que aumentaron en frecuencia y que se asociaron con periodos de disnea moderada, intercrisis y tos seca ocasional que cedieron de forma espontánea.

Dentro de sus antecedentes patológicos se destacó epilepsia diagnosticada a los 60 años de edad e hipertensión arterial en manejo irregular con fenitoína, losartán y amlodipino, sin historia de enfermedades respiratorias previas ni de insuficiencia cardiaca, ni contacto con personas sintomáticas respiratorias, sumado a exposición a humo de leña durante su infancia sin otros factores exposicionales relevantes.

Ingresó al servicio de urgencias en estado postictal tras crisis parcial compleja con generalización secundaria, polipneica con tirajes globales, saturación de oxígeno $88 \%$ ( $\mathrm{FiO}_{2}$ ambiental) TA: $137 / 83$ mm Hg FC: 74 lpm FR: 26 por min $\mathrm{T}: 36,5^{\circ} \mathrm{C}$. Cuello sin adenopatías, no ingurgitación yugular, expansión torácica adecuada, auscultación de ruidos cardiacos rítmicos, sin evidencia de soplos, pulmones con ruidos respiratorios disminuidos de predominio basal con estertores gruesos, frémito táctil y vocal conservado, con tirajes globales. Abdomen depresible, sin organomegalias ni signos de irritación peritoneal. Estable desde el punto de vista neurológico, sin alteración al examen clínico, alerta y sin focalización.

El resultado de hemograma mostró, $\mathrm{Hb}$ : 11,5; Hto: 33,19; leucocitos:16.350, N: 89,8\%, L: 8\%, eosinófilos $0,1 \%$; plaquetas: $232.000 / \mathrm{mm}^{3}$, crea-

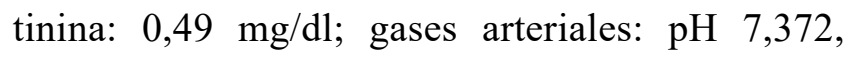
$\mathrm{pCO}_{2} 38,1 \mathrm{~mm} \mathrm{Hg}, \mathrm{pO}_{2} 70,8 \mathrm{~mm} \mathrm{Hg}, \mathrm{HCO} 3 \mathrm{~s} 21,6$ $\mathrm{mmol} / \mathrm{L}, \mathrm{EBvt}-3,1 \mathrm{mmol} / \mathrm{L}, \mathrm{PaO}_{2} / \mathrm{FiO}_{2}: 333$, sin otra alteración en los exámenes bioquímicos.

Se tomó radiografía de tórax en proyección AP que mostró un patrón de ocupación alveolar difusa, con cefalización del flujo pulmonar y silueta cardiaca aumentada de tamaño y pedículo vascular de difícil valoración por proyección anteroposterior (figura 1).

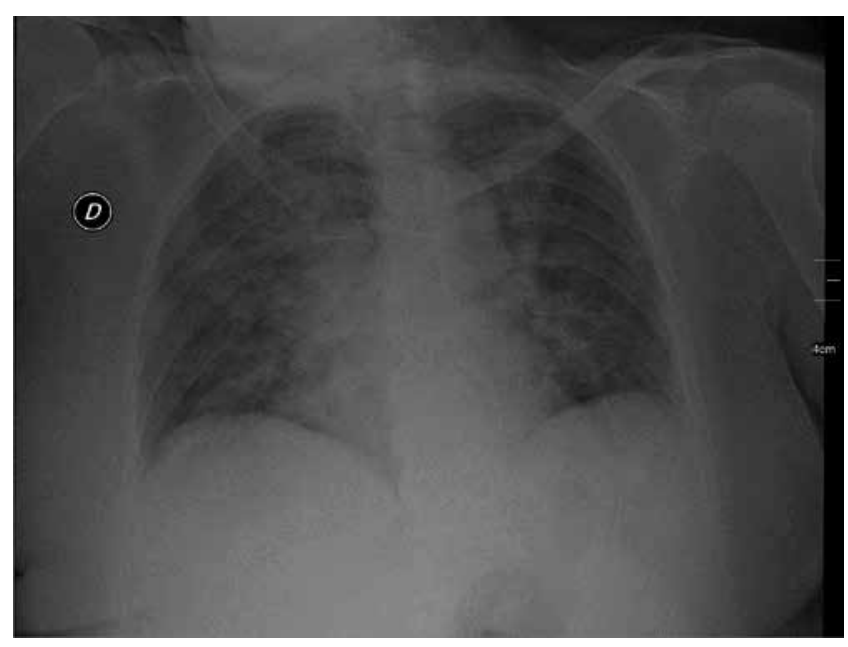

Figura 1. Radiografía de tórax donde se aprecian infiltrados de ocupación alveolar difusos. 
La paciente recibió ampicilina-sulbactam y claritromicina por sospecha de neumonía aspirativa así como ajuste de tratamiento antiepiléptico. Al cabo de 48 horas de evolución de cubrimiento antibiótico empírico, tuvo dos episodios de hemoptisis en escasa cantidad, autolimitados, sin deterioro clínico ni compromiso de su dinámica ventilatoria, con hemograma control que descartó anemización secundaria; sin embargo, dada la asociación inusual del cuadro respecto a la evolución clínica, se realizó tomografía axial computarizada de tórax que evidenció parénquima pulmonar normal, tractos de fibrosis y bandas de atelectasia en ambas bases pulmonares sin otros hallazgos relevantes (figuras 2 y 3 ).

\section{Discusión}

El edema pulmonar neurogénico es una entidad poco frecuente y de instauración rápida; corresponde al síndrome clínico caracterizado por el inicio agudo de edema pulmonar después de un daño significativo del sistema nervioso central. La falta de marcadores específicos para el diagnóstico hace que su sospecha clínica se base en desencadenantes tales como trauma cráneo-encefálico, hemorragia subaracnoidea, status epiléptico, meningitis, entre otras.

Según algunos autores el status epiléptico es la principal causa de edema pulmonar neurogénico; aparece en 1 de cada 3 pacientes principalmente durante el periodo postictal (1-3). La gravedad de presentación clínica es directamente proporcional a la afectación central. Pese a que el edema pulmonar neurogénico es de origen no cardiogénico, en el momento del abordaje diagnóstico es imperativo descartar edema pulmonar cardiogénico como etiología probable, pues este último es más frecuente.

La fisiopatología aún no es clara, pero se cree que puede deberse a una descarga simpática central, específicamente un aumento masivo en la descarga $\alpha$-adrenérgica. Algunos estudios han demostrado que el aumento de la presión arterial pulmonar es un componente esencial para el desarrollo del edema pulmonar neurogénico. Se han propuesto varios mecanismos: una teoría hemodinámica y una teoría de aumento de la permeabilidad pulmonar. La primer teoría se basa en la vasoconstricción sistémica y pulmonar tras el aumento de las catecolaminas
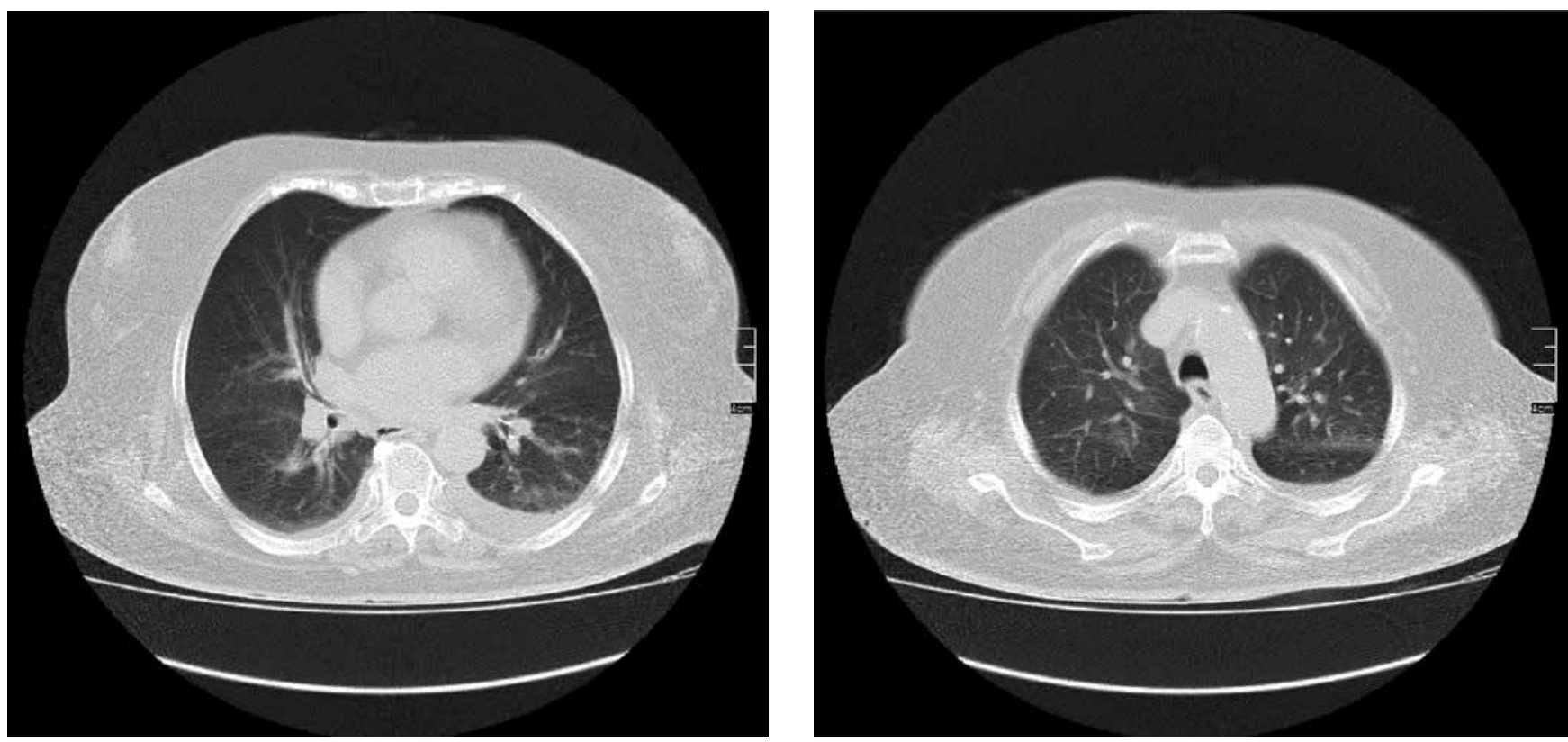

Figuras 2 y 3. Escanografía de tórax donde se evidencia parénquima pulmonar normal, tractos fibrosos y bandas de atelectasia en ambas bases pulmonares sin otros hallazgos relevantes. 
circulantes; también se ha observado que el aumento de la permeabilidad capilar pulmonar se desarrolla en el establecimiento de presiones sistémicas normales, pero bajo la influencia de una presión intracraneal elevada, lo que sugiere un mecanismo neuronal mediado para el desarrollo del edema pulmonar neurogénico (4).

En el caso expuesto, debido a la sintomatología inicial con predominio de síntomas respiratorios y quizás precedido por episodios convulsivos asociado a hallazgos radiológicos de patrón de ocupación alveolar difusa, se inició esquema empírico con ampicilina-sulbactam por sospecha de neumonía aspirativa, así como ajuste de manejo antiepiléptico con mejoría clínica sin nuevos episodios convulsivos.

En el caso actual cabe destacar que según los hallazgos en la radiografía de tórax, existen otras alternativas diagnósticas en el patrón de ocupación alveolar difusa en el abordaje inicial, tales como edema pulmonar cardiogénico, hemorragia alveolar difusa, neumonía multilobar, SDRA e incluso tuberculosis pulmonar en nuestro medio. No obstante, ante una radiografía en posición anteroposterior no se pueden tener en cuenta los debidos parámetros para evaluar la silueta cardiaca así como el pedículo vascular, los cuales son útiles en la diferenciación de edema pulmonar cardiogénico vs. no cardiogénico; adicionalmente, no existía historia previa de insuficiencia cardíaca por parámetros clínicos, electrocardiográficos o ecocardiográficos a favor del diagnóstico inicial. El ecocardiograma realizado a esta paciente no aportó datos sugestivos de insuficiencia cardiaca concomitante como un volumen indexado de la aurícula izquierda mayor a $34 \mathrm{ml} / \mathrm{m} 2$ o un índice de masa ventricular mayor a $95 \mathrm{~g} / \mathrm{m} 2$ para las mujeres o alteración en los parámetros de la función diastólica (5); y se excluyó edema pulmonar cardiogénico como primera posibilidad.

Ante la presencia probable de status convulsivo inicial con hallazgos inespecíficos de edema pulmonar de etiología no cardiogénico con la radiografía de tórax de ingreso y hemoptisis sin deterioro clínico, se vislumbró la posibilidad de edema pulmonar neurogénico confirmado mediante TAC de tórax en la cual no se apreciaron los hallazgos radiológicos iniciales, con parénquima pulmonar sin ocupación alveolar, sin evidencia de malignidad ni patología infecciosa probable y sin compromiso pleural, sumado a un ecocardiograma transtorácico normal.

No es usual pensar en edema pulmonar neurogénico, aún en presencia de alteración en el SNC, de ahí que su diagnóstico se haga por exclusión de otras patologías más frecuentes aunadas a un alto índice de sospecha clínica y al conocimiento de su fisiopatología. Davidson et al. proponen cinco criterios diagnósticos no validados en nuestro medio para edema pulmonar neurogénico:

1) Infiltrados bilaterales.

2) $\mathrm{PaO}_{2} / \mathrm{FiO}_{2}<200$.

3) Ausencia de hipertrofia ventricular izquierda.

4) Evidencia de daño al SNC.

5) Exclusión de otras causas de alteración pulmonar aguda (4).

Teniendo en cuenta el cumplimiento de cuatro criterios, la resolución al cabo de 48 horas posteriores al control completo de las crisis parciales, se destaca la presencia de un cuadro de edema pulmonar neurogénico inusual asociado a hemoptisis, cuya frecuencia de presentación poco se menciona en las actuales revisiones en la literatura.

\section{Bibliografía}

1. Simon R. Neurogenic pulmonary edema. Neurol Clin. 1993;11:309-23.

2. Colice G, Matthay M, Matthay R. Neurogenic pulmonary edema. Am Rev Resp Dis. 1984;130:941-8.

3. Simon R. Physiologic consequences of status epilepticus. Epilepsia. 1985;26(58).

4. Busl KM, Bleck TP. Neurogenic pulmonary edema. Soc Crit Care Med Wolters Kluwer Heal. 2015;43:6.

5. Ponikowski P, Voors AA, Anker SD, Bueno H, Cleland JGF, Coats AJS, et al. 2016 ESC Guidelines for the diagnosis and treatment of acute and chronic heart failure. Eur Heart J. 2016;37(27):2129-200m.

6. Davison D, Terek M, Chawla L. Neurogenic pulmonary edema. Crit Care. 2012;16(212):76-85. 
7. Lichtenberger JP, Digumarthy SR, Abbott GF, Shepard JAO, Sharma A. Diffuse pulmonary hemorrhage: Clues to the diagnosis. Curr Probl Diagn Radiol. 2014;43(3):128-39.

8. Baumann A, Audibert G, McDonnell J, Mertes PM. Neurogenic pulmonary edema. Acta Anaesthesiol Scand [Internet]. 2007;51(4):447-55. Disponible en: http://doi.wiley. com/10.1111/j.1399-6576.2007.01276.x

9. Udupi Bidkar P, Prabhakar H. Neurogenic pulmonary edema. Complicat Neuroanesthesia. 2016;43(8):181-9.
10. Díaz P, Briones J, Orellana Y, Arellano J, Faba R. Edema pulmonar neurogénico secundario a crisis epiléptica . Presentación de un caso y revisión de la literatura. Rev Chil Enfermedades Repiratorias. 2009;25:25-8.

11. Danielle L, Davison MT et al. Neurogenic pulmonary edema. Critical Care. 2012;126(1-2):76-85.

12. Lichtenberger JP, Digumarthy SR, Abbott GF, Shepard JA, Sharma A. Diffuse pulmonary hemorrhage: clues to the diagnosis. Curr Probl Diagn Radiol. 2014;43:128-39. 